\title{
Determinants of mortality of patients with COVID-19 in Wuhan, China: a case-control study
}

\author{
Jian $\mathrm{Li}^{1 \# \wedge}$, Luyu Yang ${ }^{2 \#}$, Qian Zeng ${ }^{3,4 \#}$, Qingyun $\mathrm{Li}^{5}$, Zhitao Yang ${ }^{6}$, Lizhong Han ${ }^{3,4}$, Xiaodong Huang ${ }^{7}$, \\ Erzhen Chen ${ }^{6}$
}

${ }^{1}$ Clinical Research Center, Ruijin Hospital, Shanghai Jiao Tong University School of Medicine, Shanghai, China; ${ }^{2}$ Department of ICU/Emergency, Wuhan Third Hospital, Tongren Hospital of Wuhan University, Wuhan, China; ${ }^{3}$ Department of Laboratory Medicine, Ruijin Hospital, Shanghai Jiao Tong University School of Medicine, Shanghai, China; ${ }^{4}$ Department of Clinical Microbiology, Ruijin Hospital, Shanghai Jiao Tong University School of Medicine, Shanghai, China; ${ }^{5}$ Department of Respiration and Critical Care Diseases, Shanghai Jiao Tong University School of Medicine, Shanghai, China; ${ }^{6}$ Emergency Department, Ruijin Hospital, Shanghai Jiao Tong University School of Medicine, Shanghai, China; ${ }^{7}$ Department of Gastroenterology, Wuhan Third Hospital, Tongren Hospital of Wuhan University, Wuhan, China

Contributions: (I) Conception and design: E Chen, L Han, J Li; (II) Administrative support: E Chen, X Huang; (III) Provision of study materials or patients: L Yang, Q Li, Z Yang; (IV) Collection and assembly of data: All authors; (V) Data analysis and interpretation: J Li, Q Zeng; (VI) Manuscript writing: All authors; (VII) Final approval of manuscript: All authors.

"These authors contributed equally to this work.

Correspondence to: Erzhen Chen. Emergency Department, Ruijin Hospital, Shanghai Jiao Tong University School of Medicine, Shanghai, China. Email: chen_ez@163.com; Lizhong Han. Department of Laboratory Medicine, Ruijin Hospital, Shanghai Jiao Tong University School of Medicine, Shanghai, China; Department of Clinical Microbiology, Ruijin Hospital, Shanghai Jiao Tong University School of Medicine, Shanghai, China. Email: 13916291150@163.com; Xiaodong Huang. Department of Gastroenterology, Wuhan Third Hospital, Tongren Hospital of Wuhan University, Wuhan, China. Email: hxd19681031@whu.edu.cn.

Background Coronavirus disease 2019 (COVID-19) has resulted in an overwhelmed challenge to the healthcare system worldwide.

Methods: A case-control study of COVID-19 patients in Wuhan Third Hospital was conducted. 96 deceased COVID-19 patients and 230 discharged patients were included as the case group and control group, respectively. Demographic, epidemiological, clinical and laboratory variables on admission were collected from electronic medical records. Univariate and multivariate logistic regression were adopted to investigate the independent predictors of mortality. A nomogram was formed for predicting the mortality risk.

Results: The multivariate stepwise logistic model demonstrated that age of $60+$ years $(\mathrm{OR}=4.426,95 \%$ CI: 1.955-10.019), comorbidity of cerebrovascular disease ( $\mathrm{OR}=7.084,95 \% \mathrm{CI}: 1.545-32.471$ ), white blood cell (WBC) count $>9.5 \times 10^{9} / \mathrm{L}(\mathrm{OR}=7.308,95 \% \mathrm{CI}: 1.650-32.358)$, platelet count $<125 \times 10^{9} / \mathrm{L}(\mathrm{OR}=5.128,95 \%$ CI: 2.157-12.191), aspartate aminotransferase (AST) $>40 \mathrm{U} / \mathrm{L}(\mathrm{OR}=2.554$, 95\% CI: 1.253-5.206), cystatin C $>1.1 \mathrm{mg} / \mathrm{L}$ (OR =4.132, 95\% CI: 2.118-8.059), C reactive protein (CRP) $\geq 100 \mathrm{mg} / \mathrm{L}$ (OR =2.830, 95\% CI: 1.311-6.109), creatine kinase isoenzymes (CK-MB) $>24 \mathrm{U} / \mathrm{L}(\mathrm{OR}=6.015$, 95\% CI: 2.119-17.07) and D-dimer $>5 \mu \mathrm{g} / \mathrm{L}(\mathrm{OR}=4.917,95 \% \mathrm{CI}: 1.619-14.933)$ were independent predictors of mortality of COVID-19 patients. The nomogram demonstrated a well discriminatory accuracy for mortality prediction with a C-index of 0.903.

Conclusions: The determinants identified may help to determine patients at high risk of death at an early stage and guide the optimal treatment.

Keywords: Coronavirus disease 2019 (COVID-19); mortality; determinant; case control study

Submitted Oct 25, 2020. Accepted for publication Jan 21, 2021.

doi: 10.21037/apm-20-2107

View this article at: http://dx.doi.org/10.21037/apm-20-2107

^ ORCID: 0000-0003-0901-6458. 


\section{Introduction}

Since December 2019, the pandemic of coronavirus disease 2019 (COVID-19), which originated from infection of severe acute respiratory syndrome coronavirus 2 (SARS$\mathrm{CoV}-2$ ), has spread around the world rapidly and raised serious concerns $(1,2)$. COVID-19 has given rise to an overwhelming destructive impact worldwide. As of 30 August 2020, the World Health Organization (WHO) has reported a cumulative total of nearly 25 million COVID-19 cases and 800,000 deaths globally (3). Regardless of the fact that the majority of confirmed patients were considered to be mild or moderate, $5 \%$ were critical and $14 \%$ severe on the basis of the largest survey of 72,314 patients to date (4). As with SARS-CoV (5) and Middle East Respiratory Syndrome coronavirus (MERS-CoV) (6), COVID-19 is more easily predisposed to acute respiratory distress syndrome, respiratory failure, heart failure, septic shock and even death among susceptible cases (7). Accumulated evidence from the latest studies indicates the risk factors concerning fatality of COVID-19 are heterogeneous and may include male, older age, comorbidity, smoking, elevated D-dimer level, coagulopathy, neutrophilia and organ dysfunction (8-13). However, most of those early studies came from cases series and were descriptive analysis without consideration of adequate adjustment for potential confounding factors. So the estimations of risk factors of mortality were not very robust. Studies that address these limitations are necessary in order to explore the determinants of death for COVID-19 individuals. Therefore, a casecontrol study based on COVID-19 patients from 2 campuses of a designated hospital in Wuhan, Hubei province was conducted to identify the determinants of mortality of patients with COVID-19. We present the following article in accordance with the STROBE reporting checklist (available at http://dx.doi.org/10.21037/apm-20-2107).

\section{Methods}

\section{Study design and participants}

This case-control study enrolled COVID-19 patients with definite outcomes from 2 campuses (Shouyi campus and Guanggu campus) of Wuhan Third Hospital. A total of 1,574 COVID-19 patients (1,241 in Guanggu and 334 in Shouyi) were admitted to this designated hospital from 8 January 2020 to 17 March 2020. One hundred and three of them $(6.54 \%)$ were deceased and the rest of 1471 individuals were discharged. All patients were diagnosed following the
Guideline for the Diagnosis and Treatment of COVID-19 issued by the National Health Commission of the People's Republic of China (v.5) as reported previously (14), and were laboratory-confirmed COVID-19. All deceased patients were recruited into this study as the case group. Two discharged patients to each deceased individual were randomly sampled to be used as controls. Seven deceased patients were excluded from the study owing to non-accessibility of important laboratory findings and data of comorbidities, and 230 discharged individuals were included in the study eventually. The Ethics Committees of Wuhan Third Hospitals has approved this study (No.: KY2020-007) and waived the individual informed consent due to the retrospective analysis. The study was carried out in conformity to the Declaration of Helsinki (as revised in 2013).

\section{Date collection}

Data were gathered from April 28 to May 7, 2020. The research team reviewed the medical records of COVID-19 cases. A standardized case report form was adopted to collect the data of demographics, epidemiology, symptoms and signs, laboratory and complication from electronic medical records, and two experienced doctors cross-checked all of data. In case of unavailable epidemiological data or medical history from medical records, researchers interviewed the patients or their close relatives to further ascertain. If there was a discrepancy between the medical records and the subjective description, data from medical records were used. Chest CT scan were excluded from the analysis due to massive missing of CT data at an early stage of the epidemic.

\section{Laboratory procedures}

Laboratory method for confirming SARS-CoV-2 was reported elsewhere (1). Specifically, the Chinese Center for Disease Control and Prevention (CDC) and local CDC were responsible for pathogen detecting. The real-time reverse transcription polymerase chain reaction assay (RTPCR) was adopted to detect SARS-CoV-2 in oropharyngeal swab samples. The discharge criteria involved remission of respiratory symptoms, absence of fever for 3 days and above, completely improvement in bilateral lungs by chest computed tomography (CT), and negative for 2 times in oropharyngeal swab specimens for SARS-CoV-2 RNA at least 1 day apart. Initial clinical laboratory examinations included serum biochemical tests (including liver and kidney functions), complete blood count, coagulation 
profile, myocardial enzymes and D-dimer. Frequency of examinations was placed under the discretion of doctors.

\section{Statistical analysis}

Continuous variables were expressed as median with interquartile range (IQR) and the Mann-Whitney $U$ test was adopted to compare the difference between deceased group and discharged group. Categorical variables were presented as the frequency with percentages, and were analyzed by the Pearson Chi-Square test or Fisher's Exact Test as appropriate. Potential risk factors associated with death comprised of the following features at admission: demography and epidemiology, comorbidities, symptoms and signs, together with laboratory results. To explore the determinants of COVID-19 mortality, the univariate logistic regression was used to compare the characteristics between case group and control group. A backward stepwise multivariate logistic regression analysis (both of entry and removal probability were 0.05 ) was further performed excluding variables which were not significant in univariate logistic analysis. Multiple imputation for missing data of some cases at admission was performed if missing rate were less than $20 \%$ (the maximum missing rate of variable was $16.87 \%)$. We utilized the fully conditional specification (FCS) regression methods to impute continuous variables and the discriminant function method to impute the classification variables. Continuous variables of laboratory findings were converted into categorical variables with their reference values as threshold before logistic regression model was fitted. All tests were conducted two-sided at the 5\% significance level. All above statistical analyses were performed using SAS software (v. 9.4) (SAS Institute Inc., USA). We constructed a nomogram on the basis of determinants identified in the multivariate logistic model using rms package in R Project v.3.5.2 (The $\mathrm{R}$ Foundation for Statistical Computing, Vienna, Austria. http:// www.r-project.org). The concordance index (C-index) was used to measure the predictive performance of the nomogram. The C-index fluctuated in the interval of 0.5 and 1.0 with 1.0 representing a totally correct discrimination and 0.5 denoting random chance.

\section{Results}

\section{Demographics, clinical, laboratory findings and complications}

Among 326 COVID-19 patients (274 in Guanggu campus and 52 in Shouyi campus) who had definite outcome,
96 cases had died during hospitalization, and 230 cases had recovered and been discharged. The median age of the 326 cases was 62 years (IQR 50-71; range, 25 to 98 years), and male accounted for $52.5 \%$ (Table 1); 133 (40.8\%) cases presented at least one comorbidity, with hypertension being the most common comorbidity (40.9\%), followed by diabetes $(20.1 \%)$ and cardiovascular disease $(9.0 \%)$. Only $9(3.2 \%)$ cases had a current smoking. Fever $(76.8 \%)$ and dry cough $(71.1 \%)$ were the most common symptoms on admission, followed by chest tightness (29.6\%) and sputum production (14.8\%). Median systolic blood pressure and median diastolic blood pressure on admission were 130 and $80 \mathrm{mmHg}$, respectively.

Table 1 showed the substantial differences of laboratory findings on admission between deceased group and discharged group. The results demonstrated that remarkably higher proportion of cases showing unnormal aspartate aminotransferase (AST), and significantly higher levels of $\mathrm{C}$ reactive protein (CRP), white blood cell (WBC) count, neutrophil count, neutrophil/lymphocyte ratio (NLR), globulin, total bilirubin, blood urea nitrogen, creatinine, cystatin $\mathrm{C}$, prothrombin time, creatine kinase, $\alpha$-hydroxybutyrate dehydrogenase, CK-MB and D-dimer were observed in the deceased group than in the discharged group, whereas, the platelet count, levels of lymphocyte count and thrombin time were remarkably lower in the decreased group as compared with the discharged group $(\mathrm{P}<0.05)$. There was not any statistically significant difference with regard to monocyte count, alanine aminotransferase (ALT) and total protein between the decreased group and the discharged group $(\mathrm{P}>0.05)$. Table $\mathrm{S} 1$ presented the normal ranges of laboratory indicators. Totally, the most commonly observed complication was respiratory failure $(75.5 \%)$, followed by heart failure (30.9\%), acute respiratory distress syndrome (11.3\%), acute cardiac injury (10.4\%) and acute kidney injury (9.3\%). No significant differences between deceased group and discharged group were observed with regard to all kinds of complication (Table 2). Overall, the median length of hospitalization $(\mathrm{LOH})$ was 15 days (IQR 9-23) and the median LOH of deceased group was remarkably shorter than that of discharged group $(\mathrm{P}<0.05)$.

\section{Univariate analysis of risk factors for COVID-19 mortality}

The univariate logistic analysis of possible risk factors is shown in Table 3. Demographic characters including age $\geq 60$ years old (unadjusted $\mathrm{OR}=3.846$ ) and male (unadjusted $\mathrm{OR}=1.977$ ) were associated with increased risk of death. 
Table 1 Demographic, clinical characters and laboratory parameters on admission of 326 COVID-19 patients in Wuhan, China

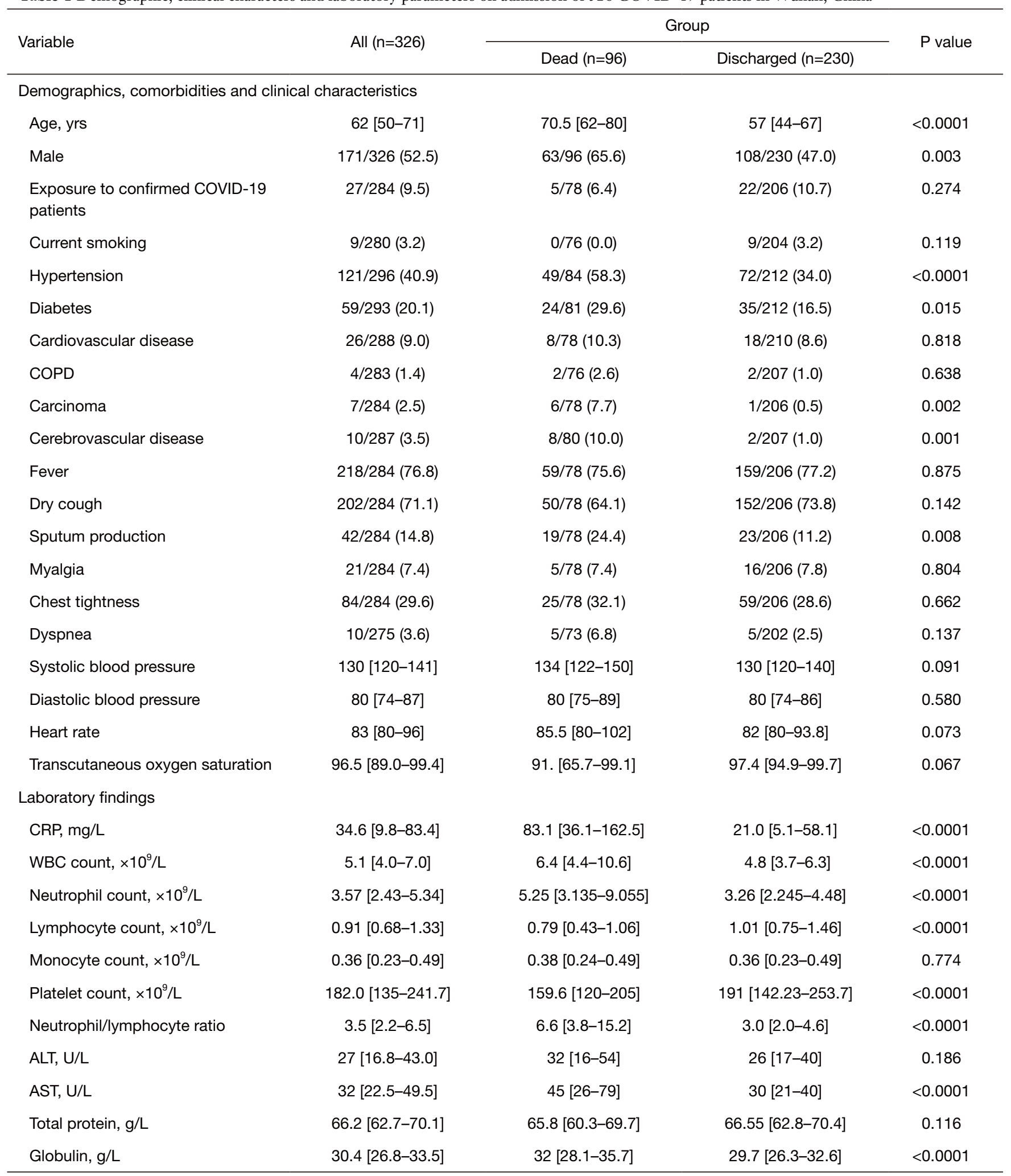

Table 1 (continued) 
Table 1 (continued)

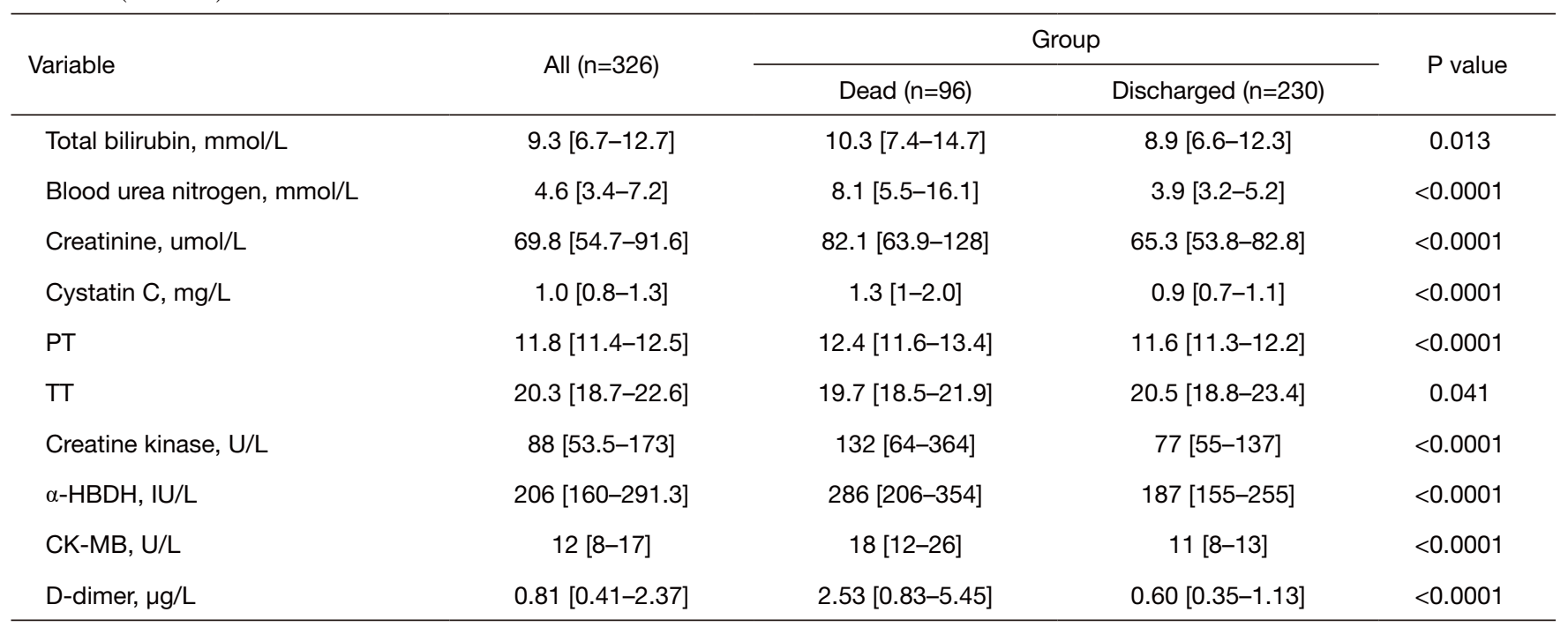

Data are presented as median [IQR] or $n /$ total (\%). $P$ value means the comparison between deceased group and discharged group. COPD, chronic obstructive pulmonary disease; CRP, C-reactive protein; WBC, white blood cell; ALT, alanine aminotransferase; AST, aspartate aminotransferase; PT, prothrombin time; TT, thrombin time; $\alpha-\mathrm{HBDH}, \alpha$-hydroxybutyrate dehydrogenase; CK-MB, creatine kinase isoenzyme.

Table 2 Complications and prognosis of 326 COVID-19 patients in Wuhan, China

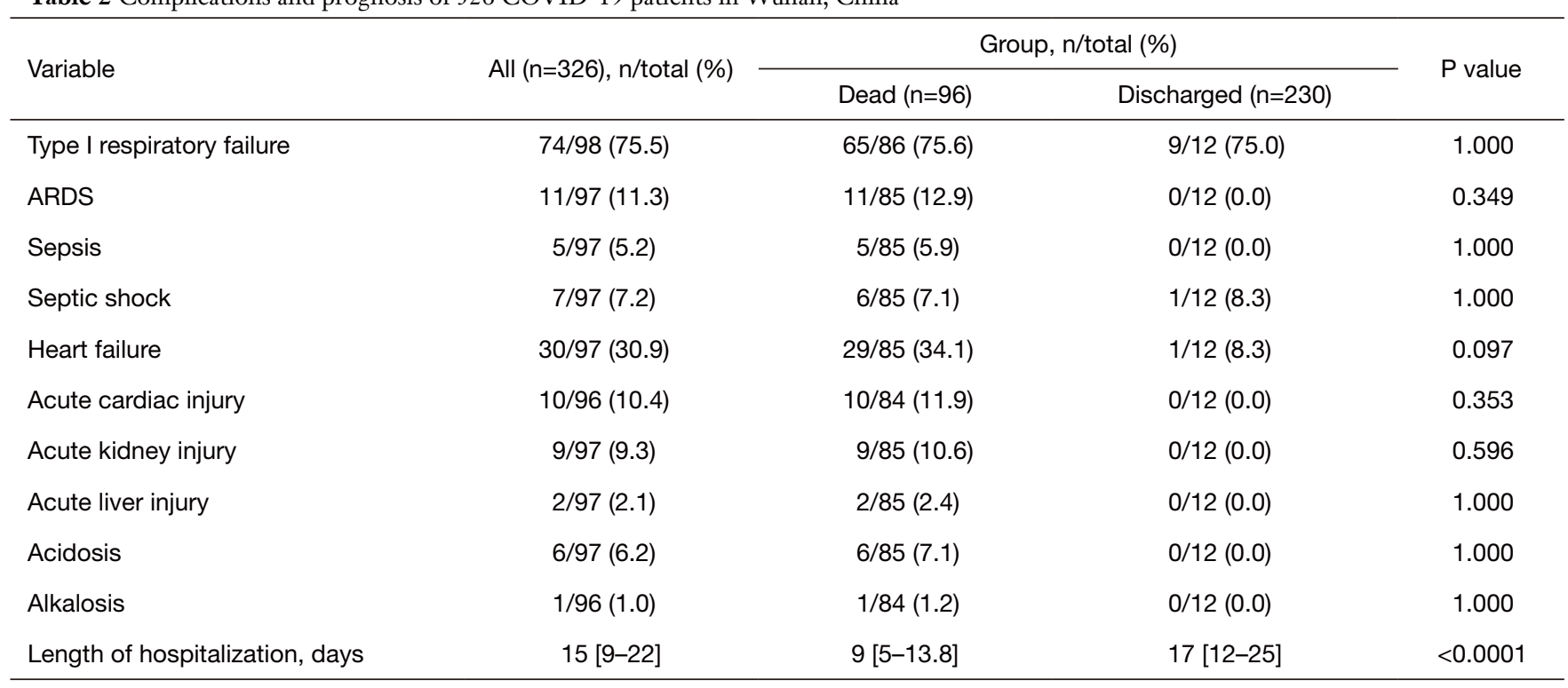

$P$ value means the comparison between deceased group and discharged group. ARDS, acute respiratory distress syndrome.

Comorbidities involving hypertension $(\mathrm{OR}=2.962)$, diabetes $(\mathrm{OR}=2.120)$, carcinoma $(\mathrm{OR}=18.007)$ and cerebrovascular disease $(\mathrm{OR}=4.655)$ appeared to be susceptible to mortality. Clinical symptoms including sputum production $(\mathrm{OR}=2.647)$ and dyspnea $(\mathrm{OR}=4.192)$, together with prolonged interval from illness onset to admission $(\mathrm{OR}=1.028)$ were found to be related to mortality. Most of laboratory findings on admission including elevated CRP (OR =6.363), elevated WBC count $(\mathrm{OR}=15.862)$, elevated NLR $(\mathrm{OR}=1.137)$, elevated ALT $(\mathrm{OR}$ $=2.256)$, elevated AST $(\mathrm{OR}=3.259)$, elevated total bilirubin 
Table 3 Univariate analysis of risk factors for mortality of COVID-19 patients in Wuhan, China

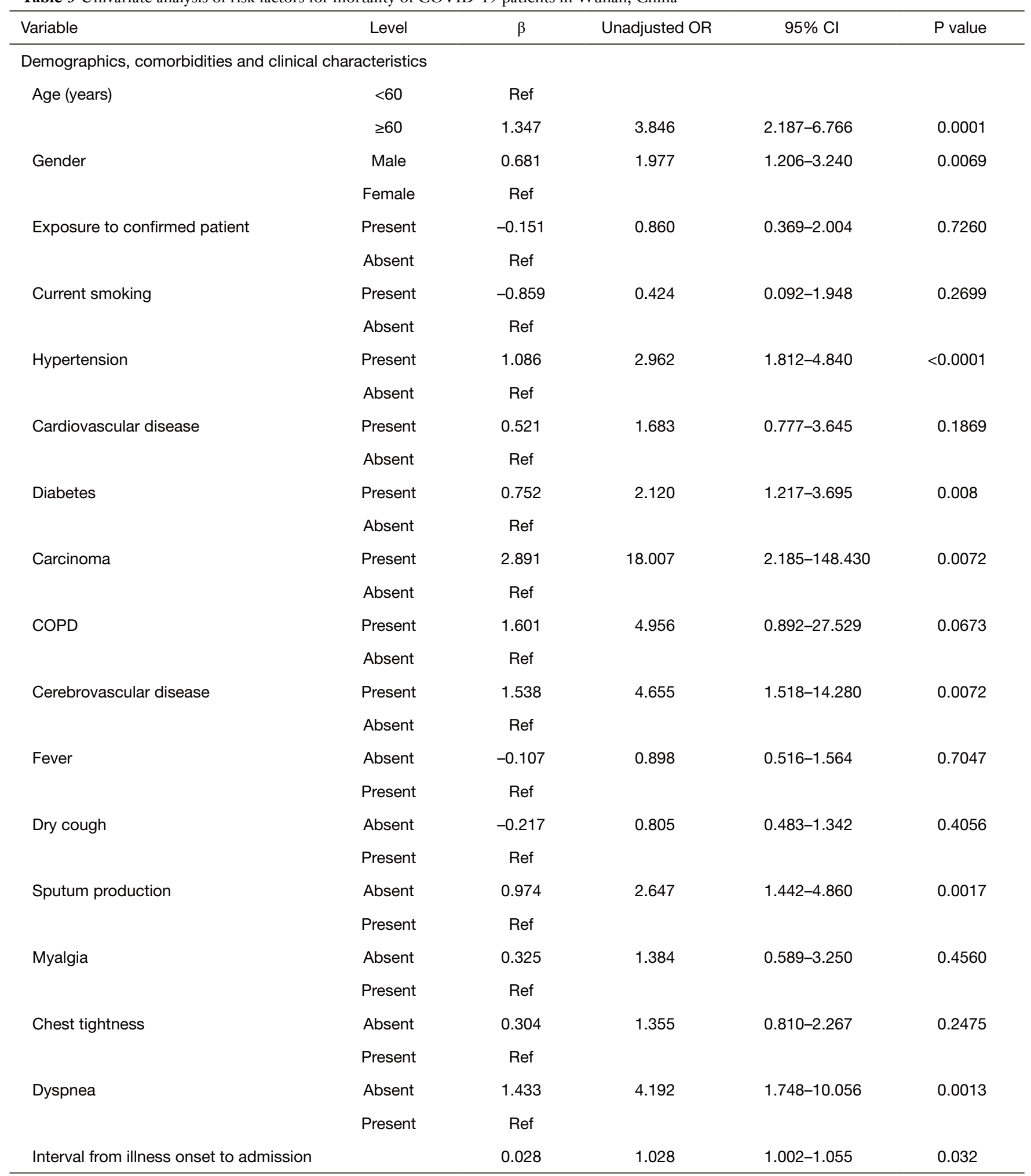

Table 3 (continued) 
Table 3 (continued)

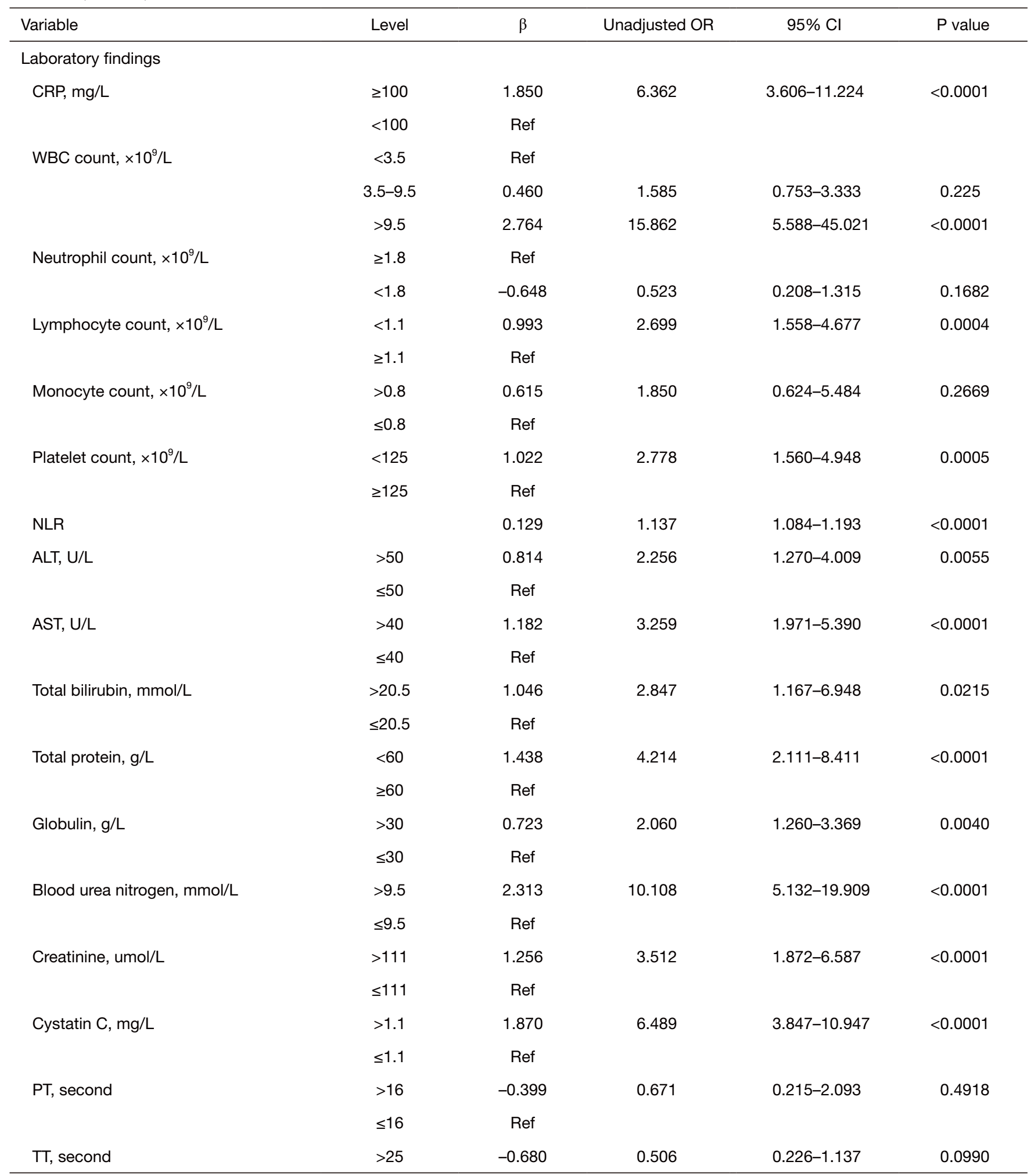

Table 3 (continued) 
Table 3 (continued)

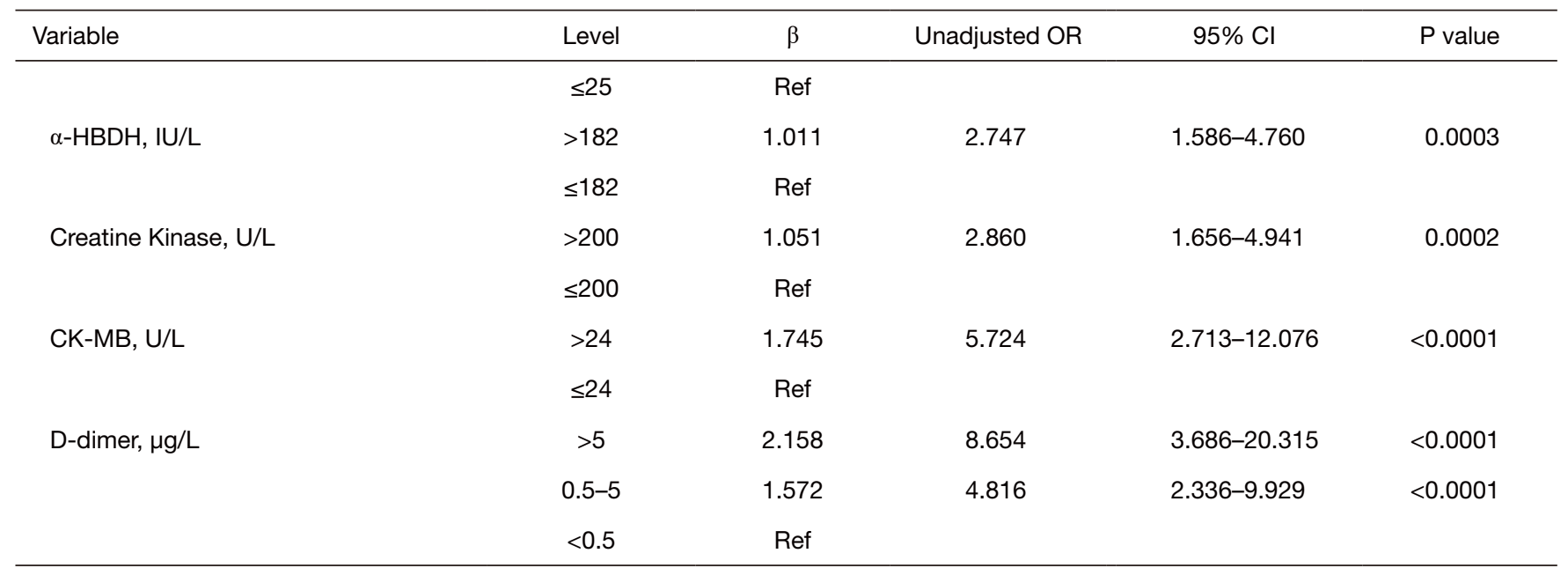

COPD, chronic obstructive pulmonary disease; CRP, C-reactive protein; WBC, white blood cell; NLR, Neutrophil/lymphocyte ratio; ALT, alanine aminotransferase; AST, aspartate aminotransferase; PT, prothrombin time; TT, thrombin time; $\alpha$-HBDH, $\alpha$-hydroxybutyrate dehydrogenase; CK-MB, creatine kinase isoenzyme.

$(\mathrm{OR}=2.847)$, elevated globulin $(\mathrm{OR}=2.060)$, elevated blood urea nitrogen ( $\mathrm{OR}=10.108)$, elevated creatinine $(\mathrm{OR}=3.512)$, elevated cystatin $\mathrm{C}(\mathrm{OR}=6.489)$, elevated $\alpha$-hydroxybutyrate dehydrogenase ( $\mathrm{OR}=2.747)$, elevated creatine kinase ( $\mathrm{OR}$ $=2.860)$, elevated CK-MB (OR =5.724), elevated D-dimer $(\mathrm{OR}=4.816$ for $0.5-5 \mu \mathrm{g} / \mathrm{L}, \mathrm{OR}=8.654$ for $>5 \mu \mathrm{g} / \mathrm{L})$, and decreased lymphocyte count $(\mathrm{OR}=2.699)$, decreased platelet count $(\mathrm{OR}=2.778)$, together with decreased total protein $(\mathrm{OR}=4.214)$ looked like to be apt to mortality. In contrast, exposure to confirmed patient, current smoking, comorbidities of cardiovascular disease, COPD, fever, cough, myalgia, chest tightness, neutrophil count, monocyte count, PT and TT were not found to be statistically different between 2 groups $(\mathrm{P}>0.05)$.

\section{Multivariate analysis of determinants of COVID-19 mortality}

Incorporating 26 significant predictors for death in univariable analysis into a multivariable logistic model with multiordinal variables converted into dummy variables, and the final model retained the 9 variables which were independently significant determinants of COVID-19 mortality (Table 4). Collinearity diagnostics of independent variables did not show multicollinearity. The results of multivariate analysis demonstrated that the mortality risk was 4.426 (95\% CI: $1.955-10.019)$ times greater among cases belonging to the age group $60+$ years as compared with those of less than 60 years. Patients with cerebrovascular disease had 7.084 (95\% CI: 1.545-32.471) times greater risk of death compared with patients without cerebrovascular disease. The risk of mortality was found higher for patients with $\mathrm{WBC}$ count $>9.5 \times 10^{9} / \mathrm{L}$ (OR $=7.308 ; 95 \%$ CI: 1.650 32.358) when compared with those having WBC count $<3.5 \times 10^{9} / \mathrm{L}$. The risk of mortality was found significantly higher for patients with lower platelet count $\left(<125 \times 10^{9} / \mathrm{L}\right)$ than those with platelet count $\geq 125 \times 10^{9} / \mathrm{L}$ with $\mathrm{OR}$ being 5.128 (95\% CI: 2.157-12.191). COVID-19 cases with AST >40 U/L on admission had a 2.554 (95\% CI: 1.253-5.206)-fold higher risk of mortality when comparison was performed with patients having normal AST level. Patients with cystatin C $>1.1 \mathrm{mg} / \mathrm{L}$ at admission had a 4.132 (95\% CI: 2.118-8.059)-fold greater risk of mortality when comparison was performed with those having cystatin $\mathrm{C}$ count $<1.1 \mathrm{mg} / \mathrm{L}$. Table 3 also showed that patients with CRP $\geq 100 \mathrm{mg} / \mathrm{L}$ and patients with CK-MB $>24 \mathrm{U} / \mathrm{L}$ had 2.830 (95\% CI: 1.311-6.109) and 6.015 (95\% CI: 2.119-17.072) times greater risk of mortality compared with those opposite, respectively. Notably, patients with elevated D-dimer were more likely to die with OR for level of between $0.5-5 \mu \mathrm{g} / \mathrm{L}$ and more than $5 \mu \mathrm{g} / \mathrm{L}$ being 2.233 (95\% CI: 0.922-5.406) and 4.917 (95\% CI: 1.619-14.933), respectively.

All the independent predictors for mortality of COVID-19 individuals in the data set were incorporated into the nomogram fitting. Figure 1 illustrates the predictive 
Table 4 Multiple logistic regression analysis of determinants for COVID-19 mortality in Wuhan

\begin{tabular}{|c|c|c|c|c|c|}
\hline Variable & Level & $\beta$ & OR & $95 \% \mathrm{Cl}$ & $P$ value \\
\hline Age (years) & $<60$ & Ref & & & \\
\hline \multirow[t]{2}{*}{ Cerebrovascular disease } & Present & 1.958 & 7.084 & $1.545-32.471$ & 0.0177 \\
\hline & Absent & Ref & & & \\
\hline \multirow{2}{*}{ WBC count $\left(\times 10^{9} / L\right)$} & $3.5-9.5$ & -0.067 & 0.936 & $0.328-2.665$ & 0.9007 \\
\hline & $>9.5$ & 1.989 & 7.308 & $1.650-32.358$ & 0.0088 \\
\hline \multirow[t]{2}{*}{ Platelet count, $\times 10^{9} / \mathrm{L}$} & $<125$ & 1.635 & 5.128 & $2.157-12.191$ & 0.0002 \\
\hline & $\geq 125$ & Ref & & & \\
\hline \multirow[t]{2}{*}{ Cystatin C, mg/L } & $>1.1$ & 1.419 & 4.132 & $2.118-8.059$ & $<0.0001$ \\
\hline & $\leq 1.1$ & Ref & & & \\
\hline \multirow[t]{2}{*}{ CRP -mg/L } & $\geq 100$ & 1.040 & 2.830 & $1.311-6.109$ & 0.0081 \\
\hline & $<100$ & Ref & & & \\
\hline \multirow[t]{2}{*}{ CK-MB, U/L } & $>24$ & 1.794 & 6.015 & $2.119-17.072$ & 0.0007 \\
\hline & $\leq 24$ & Ref & & & \\
\hline \multirow[t]{2}{*}{ D-dimer, $\mu \mathrm{g} / \mathrm{L}$} & $>5$ & 1.593 & 4.917 & $1.619-14.933$ & 0.0050 \\
\hline & $0.5-5$ & 0.803 & 2.233 & $0.922-5.406$ & 0.0750 \\
\hline
\end{tabular}

WBC, white blood cell; AST, aspartate aminotransferase; CRP, C-reactive protein; CK-MB, creatine kinase isoenzyme.

nomogram established for the mortality risk probability. A COVID-19 patient's mortality risk can simply be calculated by summing the scores for individual factor on the points scale. The nomogram demonstrated a well discriminatory accuracy for mortality prediction with a C-index of 0.903 .

\section{Discussion}

Although the COVID-19 pandemic is probable to have initiated from a zoonotic transmission event, it is wellknown that SARS-CoV-2 is highly pathogenic in humans. Mortality is the most vital problem when dealing with epidemics in the face of the gradually scarce health resources. Early identifying determinant of death of COVID-19 contributes to allocation of limited critical care and becomes the priority to minimize fatality. Nevertheless, there is a lack of reliable models for mortality predicting currently. A recent systematic review of predicting models conceived that the performance of prognostic evaluation of COVID-19 may be misleading and overoptimistic, because of the great risk of bias in vague outcome definition and subject selection (15). Regardless of the fact that there were several retrospective cohort studies evaluating mortality risk score in COVID-19 patients which aimed to earlier identify mortality risk $(13,16,17)$, they were subject to the selection bias of patients. The current case-control study was designed to determine the risk factors of mortality of COVID-19 patients in Wuhan, the source of the epidemic, in 2020. Although similar in terms of objectives to the above retrospective cohort studies, our study randomly selected the controls from the discharged patients, which was different from previous cohort studies and apparently reduced the bias in patient selection. Compare to prior similar reports, our study added some new information 


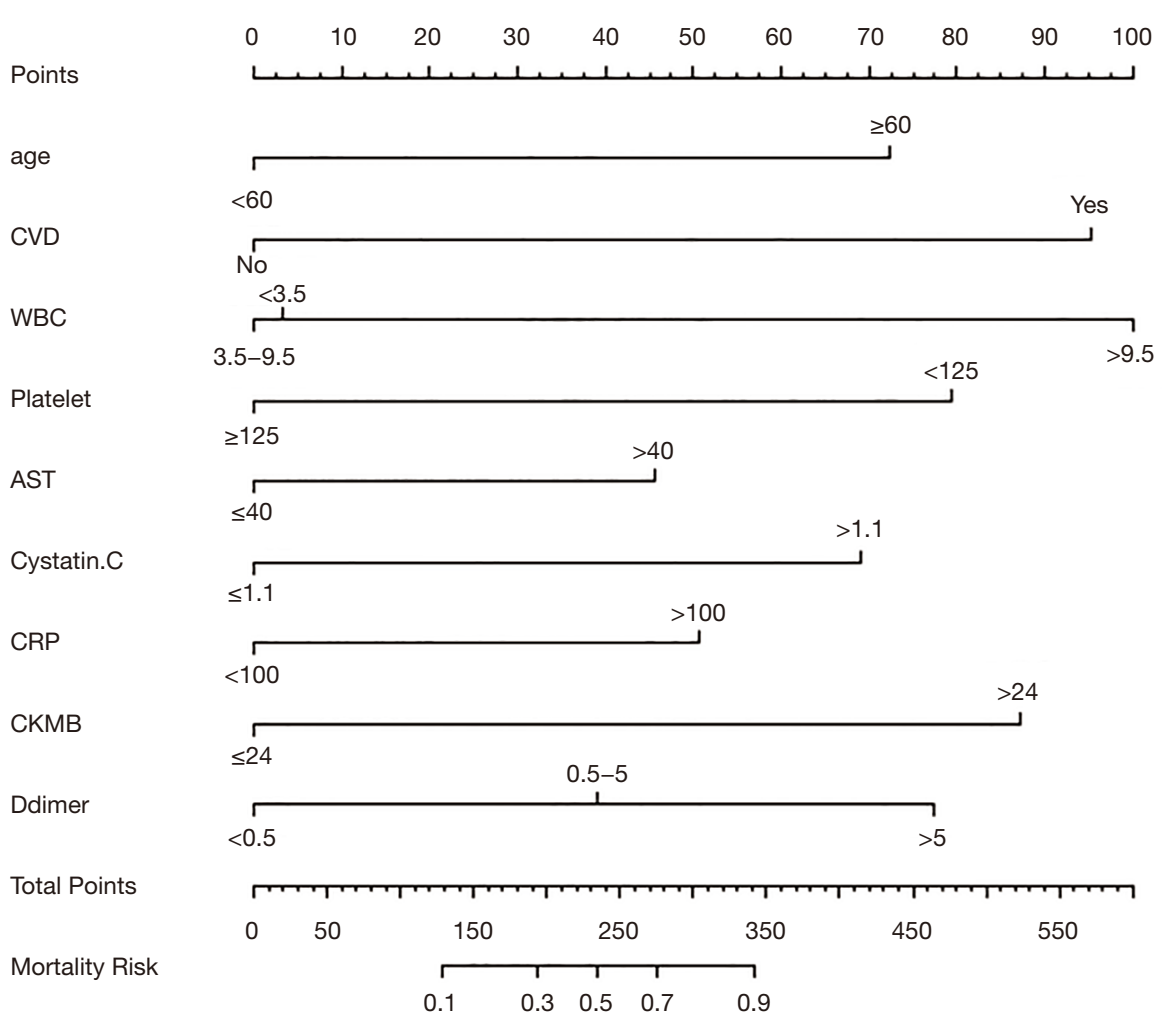

Figure 1 Nomogram for predicting mortality of COVID-19 patient. The nomogram is employed by summing the points identified on the scale for 9 factors to acquire the total points, and draw a vertical line downward to the mortality risk axis to determine the probability of death. CVD, cerebrovascular disease; WBC, white blood cell; AST, aspartate aminotransferase; CRP, C-reactive protein; CKMB, creatine kinase isoenzyme.

on independent risk factors associated with higher odds of mortality of COVID-19 inpatients such as comorbidity of cerebravascular disease, higher cystatin C and WBC count on admission, except for already described risk factors including older age, lower platelet count, higher AST, CRP, CK-MB and D-dimer on admission in the literatures.

Evidence is increasingly accumulated with respect to the determinants of COVID-19 mortality. In accordance with recent studies, advanced age has been recorded as a determinant of mortality in COVID-19 cases $(48,13,16,17)$, which is proved by our study. A deficiency in controlling for viral replication and more prolonged proinflammatory response may be caused by the excess expression of type 2 cytokines and the age-dependent defects in T-cell and B-cell function, potentially leading to a poor prognosis or even death (18). Previous studies showed COVID-19 patients combined with basic disease such as hypertension and diabetes tended to develop the adverse outcomes $(7,19)$. Less consistent with these studies, our finding suggested that commorbidity of cerebrovascular disease predisposed to death in COVID-19 patients. The SARS-CoV infection has been reported in the brains from both experimental animals and cases, where the heavily infected brainstem was observed (20). Considering the similarities between SARSCoV-2 and SARS-CoV in various aspects, cases with cerebrovascular comorbidity are more liable to be infected by SARS-CoV-2 due to low immunity and to develop cerebral complications, which is strongly associated with lethal outcome. Although leucopenia is more common in COVID-19 patients at an early stage, our study revealed that greater WBC count at admission was a dependent determinant of COVID-19 mortality. We speculated these patients with an elevated WBC count might get the second infection with bacteria, which accelerated disease progression and was more likely to induce death. It has been reported that COVID-19 had a significant impact on the liver function $(21,22)$. A study incorporating 3,428 COVID-19 cases showed that higher serum levels of ALT 
(mean difference $=7.35 \mathrm{U} / \mathrm{L}, \mathrm{P}<0.001$ ) and AST (mean difference $=8.84 \mathrm{U} / \mathrm{L}, \mathrm{P}<0.001)$ were related to a remarkable deterioration in the severity of COVID-19 and death (23). Our univariate analysis also revealed cases with elevated ALT and AST had 2.256- and 3.259-fold higher risk of death, which echoed this study. In particular, our multivariate analysis showed that AST is an independent determinant of COVID-19 mortality. As a 13 kilo Dalton proteinase inhibitor from the cystatin super family of cysteine protease inhibitors, Cystatin C plays a crucial impact in intra-cellular catabolism of peptides and proteins (24). Evidences have proven that this biomarker is a superior indicator of kidney function as compared with creatinine $(25,26)$. To date, influence of SARS-CoV-2 on cystatin C has not been reported. Our research revealed patients with higher serum cystatin $\mathrm{C}$ were prone to develop a poorer prognosis. A study investigated the potential parameters associated with imaging progression on chest CT from COVID-19 cases and found serum cystatin $\mathrm{C}$ and creatinine were significantly higher in imaging progression patients, which means a more adverse prognosis (27). Chen et al. (28) reported that the comorbidity of renal disease at admission and the progression of acute renal injury within the duration of hospitalization in cases with COVID-19 is related to inhospital mortality. As elevatory hepatic and renal injury indicators are highly associated with the exacerbation and subsequent death risk, it is strongly recommended to monitor the liver and renal function during hospitalization.

CRP is a non-specific acute-phase protein induced by IL-6 in the liver and a sensitive biomarker of tissue damage, infection and inflammation. COVID-19 cases with elevated plasma CRP in the early stage are predisposed to develop severe illness and induce the death. CRP has been proposed to be adopted as a prognostic indicator, and higher levels of CRP suggesting increased risk of disease deterioration $(29,30)$. Our result supported the studies of CRP level with COVID-19 severity elsewhere. In this study, COVID-19 patients with CRP $\geq 100 \mathrm{mg} / \mathrm{L}$ had 2.830 (95\% CI: 1.311-6.109) times greater risk of mortality, as compared with patients with $\mathrm{CRP}<100 \mathrm{mg} / \mathrm{L}$. Clinically, elevated CRP level could be early marker of nosocomial infection in COVID-19 patients that were difficult to recover, and empirical antibiotics treatment should be administered early to prevent worsened prognosis. Nearly 90 percent of pneumonia inpatients presented elevated coagulation activity, characterized by higher D-dimer level (31). As described previously, elevated D-dimer has been known as a crucial determinant of mortality in COVID-19 cases
$(7,19)$, which is proven by our research. We found patients with elevated D-dimer were associated with fatal outcome. Particularly, patients with D-dimer greater than $5 \mu \mathrm{g} / \mathrm{L}$ on admission showed a 4.917 (95\% CI: 1.619-14.933)fold higher risk of mortality when compared to those with D-dimer less than $0.5 \mu \mathrm{g} / \mathrm{L}$. Potential mechanisms include induction of procoagulant factors, systemic proinflammatory cytokine responses which are mediators of atherosclerosis directly causing plaque rupture by local inflammation, and haemodynamic changes, which contribute to ischaemia and thrombosis. Acute myocardial injury is a well-recognized cardiovascular complication among COVID-19 patients. Only $12 \%$ of survivors had heart failure as opposed to $52 \%$ of the deceased (7). Several recent researches pointed out that a greater concentration of lactate dehydrogenase (LDH), N-terminal pro B-type natriuretic peptide (NT-proBNP), CK and cTnI was related to the mortality rate of COVID-19 (32-34). Wang et al. (12) reported the median CK-MB level was remarkably lower in cases not requiring ICU care as compared with those in ICU (14 vs. $18 \mathrm{U} / \mathrm{L}, \mathrm{P}<0.01)$. Our result echoed the previous studies that the elevated CK-MB was an independent predictor of death in COVID-19 patients. Direct myocardial injury owing to the effect of systemic inflammation or viral myocarditis seems to be the most common mechanisms of acute cardiac injury. Studies on MERS-CoV and SARS-CoV had indicated that fibrinolysis and hyper coagulation could promote the likelihood of formation of microthrombus and further aggravate the risk of organ failure (35). In this study, thrombocytopenia in the early stage was determined to a determinant of COVID-19 mortality, which was consistent with other studies $(36,37)$. As well known, hyper activity of fibrinolysis can lead to the accelerating consumption of platelet. On the other hand, SARS-CoV-2 infection damaged lung tissue, causing activation, aggregation and entrapment of the platelet. This induces thrombosis on the site of pulmonary injury, which increases the depletion of platelet. Furthermore, virus infection might induce immunologic injury to platelet by bringing about auto-antibodies and immune complexes. Since reduced platelet count may aggravate the disease and be related to the adverse disease progression and even death, it is recommended to frequently measure the platelet count, leading to more effective measures much earlier. A nomogram was constructed on the basis of the independent predictors deriving from multivariate regression. The nomogram incorporating variables of governing COVID-19 mortality can serve as an easy means to evaluate the risk of 
death for COVID-19 patients and may assist physicians in decision making concerning delivery of medical care and optimal treatment. To our knowledge, this is the first report of the application of the nomogram in predicting the risk of mortality of COVID-19 patients.

Our study had several limitations. Firstly, not all laboratory indicators were detected in all cases. Although missing values were replaced through multiple imputation technique, their role in predicting mortality might be underestimated. Secondly, owing to massive missing of chest CT data at an early stage of the epidemic, this study could not evaluate the forecasting role of chest $\mathrm{CT}$ abnormalities. Its role for predicting mortality risk of COVID-19 has been proven by previous study (13). Thirdly, regardless of the fact that the subjects came from 2 campuses of a designated hospital, generalisability of the study results is probably restricted by the sample size, and is necessary to be validated on the basis of much larger patients.

\section{Conclusions}

In this research, we identified that older age, comorbidity of cerebrovascular disease, higher WBC count, AST, cystatin C, CRP, CK-MB, D-dimer and lower platelet count on admission as the independent determinants to predict the mortality risk in COVID-19 cases. Considering the ongoing worldwide pandemic of COVID-19, the current research will be helpful to identify patients at great risk of death at an early stage and guide the clinical treatment.

\section{Acknowledgments}

We acknowledge all health-care workers involved in the diagnosis and treatment of patients in study site, and we would like to thank Dr. Binxun Liao, Dr. Yueyuan Wang, Dr. Zhipeng Li for helping data cleaning. We also acknowledge Prof. Genyan Yang (Centers for Disease Control and Prevention, Atlanta, GA, USA) for language editing and critical review of the manuscript.

Funding: This study was funded by grants from Shanghai Municipal Science \& Technology Commission Key Scientific and Technological Innovation Action Plan (18411950900), National Nature Science Funds of China (81772107) and Shanghai Shenkang Hospital Development Center Clinical Science and Technology Innovation Project (SHDC12017116).

\section{Footnote}

Reporting Checklist: The authors have completed the
STROBE reporting checklist. Available at http://dx.doi. org/10.21037/apm-20-2107

Data Sharing Statement: Available at http://dx.doi. org/10.21037/apm-20-2107

Conflicts of Interest: All authors have completed the ICMJE uniform disclosure form (available at http://dx.doi. org/10.21037/apm-20-2107). The authors have no conflicts of interest to declare.

Ethical Statement: The authors are accountable for all aspects of the work in ensuring that questions related to the accuracy or integrity of any part of the work are appropriately investigated and resolved. The Ethics Committees of Wuhan Third Hospitals has approved this study (No.: KY2020-007) and waived the individual informed consent due to the retrospective analysis. The study was carried out in conformity to the Declaration of Helsinki (as revised in 2013).

Open Access Statement: This is an Open Access article distributed in accordance with the Creative Commons Attribution-NonCommercial-NoDerivs 4.0 International License (CC BY-NC-ND 4.0), which permits the noncommercial replication and distribution of the article with the strict proviso that no changes or edits are made and the original work is properly cited (including links to both the formal publication through the relevant DOI and the license). See: https://creativecommons.org/licenses/by-nc-nd/4.0/.

\section{References}

1. Huang C, Wang Y, Li X, et al. Clinical features of patients infected with 2019 novel coronavirus in Wuhan, China. Lancet 2020;395:497-506.

2. Chen N, Zhou M, Dong X, et al. Epidemiological and clinical characteristics of 99 cases of 2019 novel coronavirus pneumonia in Wuhan, China:a descriptive study. Lancet 2020;395:507-13.

3. World Health Organization. Coronavirus disease (COVID-19) weekly epidemiological update. 2020. Accessed 31 August 2020. Available online: https:// www.who.int/docs/default-source/coronaviruse/ situation-reports/20200831-weekly-epi-update-3. pdf?sfvrsn=d7032a2a_4

4. Wu Z, McGoogan JM. Characteristics of and Important Lessons From the Coronavirus Disease 2019 (COVID-19) 
Outbreak in China: Summary of a Report of 72314 Cases From the Chinese Center for Disease Control and Prevention. JAMA 2020;323:1239-42.

5. Booth CM, Matukas LM, Tomlinson GA, et al. Clinical features and short-term outcomes of 144 patients with SARS in the greater Toronto area. JAMA 2003;289:2801-9.

6. Rahman A, Sarkar A. Risk factors for fatal Middle East Respiratory Syndrome coronavirus infections in Saudi Arabia: analysis of the WHO line list, 2013-2018. Am J Public Health 2019;109:1288-93.

7. Zhou F, Yu T, Du RH, et al. Clinical course and risk factors for mortality of adult inpatients with COVID-19 in Wuhan, China: a retrospective cohort study. Lancet 2020;395:1054-62.

8. Wu C, Chen X, Cai Y, et al. Risk factors associated with acute respiratory distress syndrome and death in patients with corona virus disease 2019 pneumonia in Wuhan, China. JAMA Intern Med 2020;180:934-43. Erratum in: JAMA Intern Med 2020;180:1031.

9. Livingston E, Bucher K. Coronavirus Disease 2019 (COVID-19) in Italy. JAMA 2020;323:1335.

10. Guan WJ, Liang WH, Zhao Y, et al. Comorbidity and its impact on 1590 patients with COVID-19 in China: a nationwide analysis. Eur Respir J 2020;55:2000547.

11. Huang R, Zhu L, Xue LY, et al. Clinical findings of patients with coronavirus disease 2019 in Jiangsu Province, China: a retrospective, multi-center study. PLoS Negl Trop Dis 2020;14:e0008280.

12. Wang D, Hu B, Hu C, et al. Clinical Characteristics of 138 Hospitalized Patients With 2019 Novel CoronavirusInfected Pneumonia in Wuhan, China. JAMA 2020;323:1061-9.

13. Liang W, Liang H, Ou L, et al. Development and Validation of a Clinical Risk Score to Predict the Occurrence of Critical Illness in Hospitalized Patients With COVID-19. JAMA Intern Med 2020;180:1081-9.

14. Feng Y, Ling Y, Bai T, et al. COVID-19 with Different Severity: A Multi-center Study of Clinical Features. Am J Respir Crit Care Med 2020;201:1380-8.

15. Wynants L, Van Calster B, Collins GS, et al. Prediction models for diagnosis and prognosis of covid-19 infection: systematic review and critical appraisal. BMJ 2020;369:m1328.

16. Fumagalli C, Rozzini R, Vannini M, et al. Clinical risk score to predict in-hospital mortality in COVID-19 patients: a retrospective cohort study. BMJ Open 2020;10:e040729.
17. Altschul DJ, Unda SR, Benton J, et al. A novel severity score to predict inpatient mortality in COVID-19 patients. Sci Rep 2020;10:16726.

18. Opal SM, Girard TD, Ely EW. The immunopathogenesis of sepsis in elderly patients. Clin Infect Dis 2005;41:S504-12.

19. Zheng Z, Peng F, Xu B, et al. Risk factors of critical \& mortal COVID-19 cases: A systematic literature review and meta-analysis. J Infect 2020;81:e16-25.

20. Li YC, Bai WZ, Hashikaw T. The neuroinvasive potential of SARS-CoV2 may play a role in the respiratory failure of COVID-19 patients. J Med Virol 2020;92:552-5.

21. Zhang Y, Zheng L, Liu L, et al. Liver impairment in COVID-19 patients: a retrospective analysis of 115 cases from a single centre in Wuhan city, China. Liver Int 2020;40:2095-103.

22. Lei F, Liu YM, Zhou F, et al. Longitudinal association between markers of liver injury and mortality in COVID-19 in China. Hepatology 2020;72:389-98.

23. Parohan M, Yaghoubi S, Seraj A. Liver injury is associated with severe coronavirus disease 2019 (COVID-19) infection: a systematic review and meta-analysis of retrospective studies. Hepatol Res 2020;50:924-35.

24. Nakhjavan-Shahraki B, Yousefifard M, Ataei N, et al. Accuracy of cystatin $\mathrm{C}$ in prediction of acute kidney injury in children; serum or urine levels: which one works better? A systematic review and meta-analysis. BMC Nephrol 2017;18:120.

25. Dharnidharka VR, Kwon C, Stevens G. Serum systatin $\mathrm{C}$ is superior to serum creatinine as a marker of kidney function: a meta-analysis. Am J Kidney Dis 2002;40:221-6.

26. Roos JF, Doust J, Tett SE, et al. Diagnostic accuracy of cystatin $\mathrm{C}$ compared to serum creatinine for the estimation of renal dysfunction in adults and children- a metaanalysis. Clin Biochem 2007;40:383-91.

27. Yang Z, Shi J, He Z, et al. Predictors for imaging progression on chest CT from coronavirus disease 2019 (COVID-19) patients. Aging (Albany NY) 2020;12:6037-48.

28. Cheng Y, Luo R, Wang $\mathrm{K}$, et al. Kidney disease is associated with in-hospital Death of patients with COVID-19. Kidney Int 2020;97:829-38.

29. Chen W, Zheng KI, Liu SD, et al. Plasma CRP level is positively associated with the severity of COVID-19. Ann Clin Microbiol Antimicrob 2020;19:18.

30. Liu F, Li L, Xu MD, et al. Prognostic value of interleukin-6, C-reactive protein, and procalcitonin in patients with COVID-19. J Clin Virol 2020;127:104370. 
31. Milbrandt EB, Reader MC, Lee M, et al. Prevalence and significance of coagulation abnormalities in communityacquired pneumonia. Mol Med 2009;15:438-45.

32. Inciardi RM, Adamo M, Lupi L, et al. Characteristics and outcomes of patients hospitalized for COVID-19 and cardiac disease in northern Italy. Eur Heart J 2020;41:1821-9.

33. Gaze DC. Clinical Utility of Cardiac Troponin Measurement in COVID-19 Infection. Ann Clin Biochem 2020;57:202-5.

34. Ruan Q, Yang K, Wang W, et al. Clinical predictors of mortality due to COVID-19 based on an analysis of data of 150 patients from Wuhan, China. Intensive Care Med
2020;46:846-8.

35. So LK, Lau AC, Yam LY, et al. Development of a standard treatment protocol for severe acute respiratory syndrome. Lancet 2003;361:1615-7.

36. Lippi G, Plebani M, Henry BM. Thrombocytopenia is associated with severe cornonvirus disease 2019(COVID-19) infections: A meta-analysis. Clin Chim Acta 2020;506:145-8.

37. Zhao X, Wang K, Zuo P, et al. Early decrease in blood platelet count is associated with poor prognosis in COVID-19 patients-indications for predictive, preventive, and personalized medical approach. EPMA J 2020;11:1-7.

Cite this article as: Li J, Yang L, Zeng Q, Li Q, Yang Z, Han L, Huang X, Chen E. Determinants of mortality of patients with COVID-19 in Wuhan, China: a case-control study. Ann Palliat Med 2021;10(4):3937-3950. doi: 10.21037/apm-20-2107 
Supplementary

Table S1 Reference range of laboratory indicators

\begin{tabular}{|c|c|c|}
\hline Indicator & Unit & Reference range \\
\hline C-reactive protein & $\mathrm{mg} / \mathrm{L}$ & $<5.00$ \\
\hline White blood cell count & $\times 10^{9} / \mathrm{L}$ & $3.50-9.50$ \\
\hline Neutrophil count & $\times 10^{9} / \mathrm{L}$ & $1.80-6.30$ \\
\hline Lymphocyte count & $\times 10^{9} / \mathrm{L}$ & $1.10-3.20$ \\
\hline Monocyte count & $\times 10^{9} / \mathrm{L}$ & $0.12-0.80$ \\
\hline Platelet count & $\times 10^{9} / \mathrm{L}$ & $125-350$ \\
\hline ALT & $\mathrm{U} / \mathrm{L}$ & $9.0-50.0$ \\
\hline AST & $\mathrm{U} / \mathrm{L}$ & $15.0-40.0$ \\
\hline Total bilirubin & umol/L & $3.40-20.50$ \\
\hline Total protein & $g / L$ & $60.0-80.0$ \\
\hline Globulin & $g / L$ & $20.0-30.0$ \\
\hline Blood urea nitrogen & $\mathrm{mmol} / \mathrm{L}$ & $3.60-9.50$ \\
\hline Creatinine & umol/L & $57.00-111.00$ \\
\hline Cystatin C & $\mathrm{mg} / \mathrm{L}$ & $0.5-1.1$ \\
\hline PT & second & $11-16$ \\
\hline$\pi$ & second & $18-25$ \\
\hline$\alpha-\mathrm{HBDH}$ & IU/L & $90-182$ \\
\hline Creatine Kinase & $\mathrm{U} / \mathrm{L}$ & $30.00-200.00$ \\
\hline CK-MB & $\mathrm{U} / \mathrm{L}$ & $<24$ \\
\hline D-dimer & $\mu g / L$ & $<0.50$ \\
\hline
\end{tabular}

ALT, alanine aminotransferase; AST, aspartate aminotransferase; PT, prothrombin time; TT, thrombin time; $\alpha$-HBDH, $\alpha$-hydroxybutyrate dehydrogenase; CK-MB, creatine kinase isoenzyme. 